\title{
Numerical simulation of particle dispersion in a pre-processing of a static culture
}

\author{
Yoshiki Kanemaru ${ }^{1}$, Atsushi Sekimoto ${ }^{1}$, Yasunori Okano ${ }^{1, *}$, Kei Kanie $^{2}$, Ryuji Kato $^{2}$, and Masahiro Kino-oka ${ }^{3}$ \\ ${ }^{1}$ Department of Materials Engineering Science, Osaka University, 1-3 Machikaneyama, Toyonaka, Osaka 560-8531, Japan \\ ${ }^{2}$ Department of Basic Medicinal Science, Nagoya University, Furocho, Chiusa-ku, Aichi 464-8601, Japan \\ ${ }^{3}$ Department of Biotechnology, Osaka University, 1-2 Yamadaoka, Suita, Osaka 565-0871, Japan
}

\begin{abstract}
For producing high-quality induced pluripotent stem (iPS) cells in a static culture, initial placement of cells is one of the most important factors. Dense distribution of cells increases the risk of cell death. Thus, the cells need to be uniformly distributed during the preprocess of a static culture. This process depends on the operator's experiences and has not been standardized. In this study, the authors have performed numerical simulations to investigate the efficient of cell dispersion by using OpenFOAM. The numerical domain is a square-shaped dish. Two shaking methods, one-direction and multi-direction reciprocal shaking, were considered and calculations were conducted under five oscillation frequencies. The cell colony was assumed as a solid spherical particle. The initial particles were densely positioned at the center. The numerical result showed that the multi-direction reciprocal shaking was more effective to disperse the particles than the one-direction reciprocal shaking. In addition, almost all particles at low frequency sank to the bottom and hardly dispersed. These results indicate that strong fluctuations can lift particles from the bottom, and frequent change of flow direction makes distribution area wide.
\end{abstract}

\section{Introduction}

Induced Pluripotent Stem (iPS) cells have the ability to differentiate into a lot of tissue cells [1-2]. Compared with Embryonic Stem (ES) cells [3] that have similar characteristics to iPS cells, iPS cells do not have ethical and immune problems because iPS cells can be produced from patient's somatic cells, and they are expected to contribute to regenerative medicine and drug development. However, culturing iPS cells needs skillful technicians, so this process depends on operator's experience, and a large quantity of cells cannot be cultured at one operation. Therefore, development of the automation and mass production method is required. Our research group had previously focused on suspension culture and investigated shear stress acting on the cell colony [4]. In this study, the authors focused on static culture with the aim to automate the culturing operation.

In a static culture, initial placement of the cells is one of the most important factors to produce high-quality cells. Cells have to be positioned uniformly in a culture dish because dense distribution of cells increases of the risk of cell death. To obtain optimal shaking method, the authors have to quantify the particle distribution and find out exactly what happens in a culture dish. However, there are few reports that investigate cell seeding process by using numerical simulation. In this study, the authors have performed numerical simulations to investigate the cell dispersion in a bioreactor with three horizontal reciprocating movements.

\section{Numerical methods}

In this study, the following assumptions were considered to represent the cell culture.

(1) The fluid is Newtonian and incompressible.

(2) Any chemical nor biological reaction does not occur and temperature is uniform in both the solid and the liquid phases.

(3) A single cell colony is represented by a solid spherical particle, which does not change in size.

(4) The collision of the particles with the side and the bottom wall is perfectly elastic.

(5) The collision between particles is neglected because the number of seeded cells is small in an actual static culture.

The numerical domain is shown in Fig. 1. It was assumed that the container is a square-shaped dish. The bottom face was $74 \mathrm{~mm}$ on each side, and the height was $37 \mathrm{~mm}$. The initial height of culture medium was set to 3 $\mathrm{mm}$.

Three shaking methods, one-direction reciprocal shaking (ORS), multi-direction reciprocal shaking which changes shaking direction by 90 degrees every 1 reciprocation (MRS1) and multi-direction reciprocal shaking which changes shaking direction by 90 degrees every 2 reciprocations (MRS2), were considered and calculations were conducted under five shaking frequencies. All shaking methods were stopped shaking after 8 reciprocations. The particle distribution was evaluated when a sufficient time has elapsed after the shaking was stopped.

* Corresponding author: okano@ cheng.es.osaka-u.ac.jp 


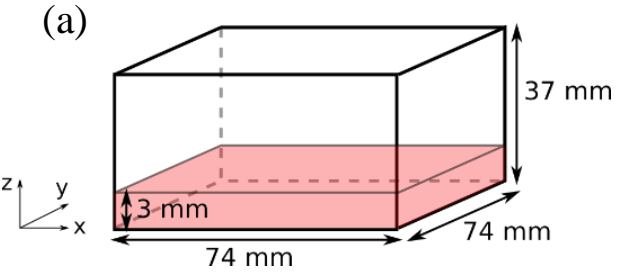

(b)

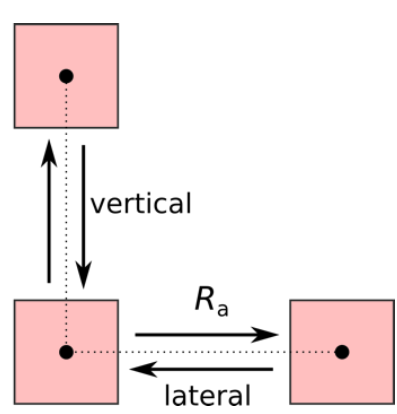

Fig. 1. (a) Schematics of a numerical domain, and (b) top view of the motion of the dish.

To calculate the motion of fluid and particle behavior, the authors have developed a numerical model by coupling the Computational Fluid Dynamics (CFD) with the Discrete Element Method (DEM) [5-6]. CFD expresses the culture medium behavior by solving continuity and Navier-Stokes equations. On the other hand, DEM expresses the particle behavior by solving Newton's second law of motion. An one-way (fluid to particle) method is used and the effect of particle to fluid is neglected because the concentration of particles is low. In addition, Volume of Fluid (VOF) method [7] is used to calculate two-phase flow.

Governing equations are continuous and NavierStokes equations shown in the following. The surface tension term was added to Navier-Stokes equation to calculate the two-phase flow ${ }^{[8]}$.

$$
\nabla \cdot v_{\mathrm{f}}=0
$$

$$
\begin{aligned}
\frac{\partial}{\partial t}\left(\rho_{\mathrm{f}} v_{\mathrm{f}}\right)+\nabla \cdot\left(\rho_{\mathrm{f}} v_{\mathrm{f}} v_{\mathrm{f}}\right) & =-\nabla p+\nabla \cdot\left(\mu_{\mathrm{f}} \nabla v_{\mathrm{f}}\right)+\rho_{\mathrm{f}} \boldsymbol{g} \\
& -m_{\mathrm{s}} \frac{d \boldsymbol{U}}{d t}+\sigma \kappa \boldsymbol{n} \delta
\end{aligned}
$$

Particle behavior was calculated by adopting DEM. The governing equation is Newton's second law of motion.

$$
\begin{gathered}
m_{\mathrm{s}} \frac{d \boldsymbol{v}_{\mathrm{s}}}{d t}=\boldsymbol{F}_{\mathrm{D}}+\boldsymbol{F}_{\mathrm{g}}-m_{\mathrm{s}} \frac{d \boldsymbol{U}}{d t} \\
\boldsymbol{F}_{\mathrm{D}}=C_{\mathrm{D}} A_{\mathrm{p}} \frac{\rho_{\mathrm{f}}\left|\boldsymbol{v}_{\mathrm{f}}-\boldsymbol{v}_{\mathrm{s}}\right|^{2}}{2} \frac{\boldsymbol{v}_{\mathrm{f}}-\boldsymbol{v}_{\mathrm{s}}}{\left|\boldsymbol{v}_{\mathrm{f}}-\boldsymbol{v}_{\mathrm{s}}\right|}
\end{gathered}
$$

$$
\begin{aligned}
& C_{\mathrm{D}}= \begin{cases}\frac{24}{R e_{\mathrm{p}}}\left(1+\frac{1}{6} R e_{\mathrm{p}}^{\frac{2}{3}}\right) & R e_{\mathrm{p}} \leq 1000 \\
0.424 & R e_{\mathrm{p}} \geq 1000\end{cases} \\
& R e_{\mathrm{p}}=\frac{\rho_{\mathrm{f}}\left|v_{\mathrm{f}}-v_{\mathrm{s}}\right| d_{\mathrm{s}}}{\mu_{\mathrm{f}}} \\
& \boldsymbol{F}_{\mathrm{g}}=m_{\mathrm{s}} \boldsymbol{g}\left(1-\frac{\rho_{\mathrm{f}}}{\rho_{\mathrm{s}}}\right) \\
& \boldsymbol{U}=\left(\begin{array}{l}
\frac{d r}{d x} \\
\frac{d r}{d y}
\end{array}\right)=\left(\begin{array}{c}
-R_{\mathrm{a}} \omega \sin \omega t \\
0
\end{array}\right) \\
& \boldsymbol{U}=\left(\begin{array}{l}
\frac{d r}{d x} \\
\frac{d r}{d y}
\end{array}\right)=\left(\begin{array}{c}
-R_{\mathrm{a}} \omega \sin \omega t \\
0 \\
\uparrow \\
0 \\
-R_{\mathrm{a}} \omega \sin \omega t
\end{array}\right)
\end{aligned}
$$

Here, the drag coefficient, $C_{\mathrm{D}}$, is a function of the particle Reynolds number, $R e_{\mathrm{p}}$, which is calculated by the velocity difference between the fluid and the particle. $\boldsymbol{U}$ indicates the velocity of the reciprocal shaking dish. In the ORS, a dish reciprocally moves on the same straight line. Two kinds of MRS is considered, one changes shaking direction every 1 reciprocation (MRS1) and the other changes shaking direction every 2 reciprocations (MRS2). The motion and the dish was represented by introducing external force in the Eq. 2 and Eq. 3. The amplitude, $R_{\mathrm{a}}$, is $34 \mathrm{~mm}$.

VOF method was used to calculate gas-liquid interface. Fluid and gas phases are defined as $\alpha=1$ and $\alpha=0$, respectively. Therefore, gas-liquid interface is $0<\alpha<1$. The advection equation of fluid fraction, $\alpha$, is

$$
\begin{gathered}
\frac{\partial \alpha}{\partial t}+\nabla \cdot(\alpha v)+\nabla \cdot\left((1-\alpha) \alpha v_{\mathrm{r}}\right)=0 \\
\boldsymbol{v}=\alpha \boldsymbol{v}_{\mathrm{f}}+(1-\alpha) v_{\mathrm{f}}
\end{gathered}
$$

The densities of water and air are 996 and $1 \mathrm{~kg} \cdot \mathrm{m}^{-3}$, respectively. The kinematic viscosity is $1.11 \times 10^{-6} \mathrm{~m}^{2} \cdot \mathrm{s}^{-1}$. The surface tension of water is $72.8 \times 10^{-3} \mathrm{~N} \cdot \mathrm{m}^{-1}$.

The particle diameter, $d_{s}$, is $0.3 \mathrm{~mm}$, and the number of particles was 990 . The density of the particles was set to $1075 \mathrm{~kg} \cdot \mathrm{m}^{-3}$. Hence, the specific gravity of the particles was 1.08. Initially, the particles were densely positioned at the bottom center.

The governing equations were discretized by the finite volume method. A second order linear interpolation scheme was used to calculate particle velocity, and the first-order implicit Euler method was applied for the integration in time. These calculations were conducted in a framework of OpenFOAM, which is open source CFD tool box [8]. 


\section{Results and discussion}

Numerical results were visualized as a top view of particle distribution at 6 seconds after the shaking was stopped. The domain was divided into $20 \times 20$ small bins, and number of particles were counted.

\subsection{Effect of shaking method}

First, the authors investigated the effect of the shaking methods. The shaking frequency $(f=\omega / 2 \pi)$ of all cases was set to $1 \mathrm{~s}^{-1}$. The particle distribution of ORS, MRS1, and MRS2 are shown in Fig. 2. MRS1 shows the highest particle dispersion. On the other hand, particles were distributed in a single line in the ORS method. These results indicate that frequent change of flow direction widens distribution area.

(a) MRS1

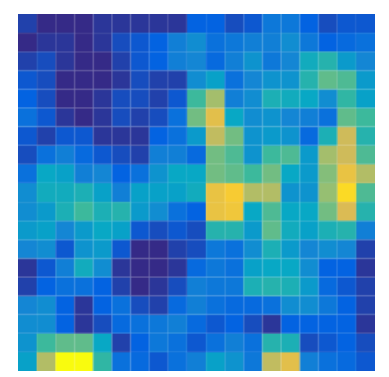

(b) ORS

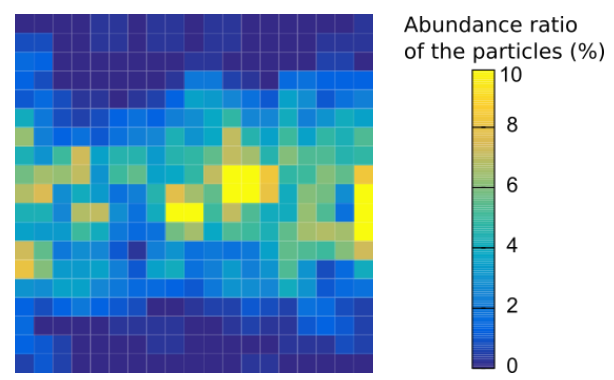

(c) MRS2

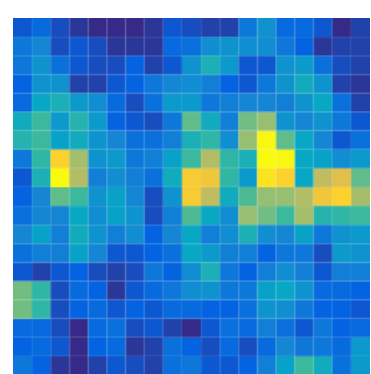

Fig. 2 Top view of particle distribution in three shaking methods $\left(f=1 \mathrm{~s}^{-1}\right)$

\subsection{Effect of shaking frequency}

Next, the authors investigated the effect of the shaking frequency. The numerical analysis was conducted by changing the shaking frequency $(f=\omega / 2 \pi)$ from 0.5 $\mathrm{s}^{-1}$ to $2 \mathrm{~s}^{-1}$, and their distributions are shown in Fig. 3 . The MRS1 method was used as a shaking method. As the shaking frequency increases, the particles that were initially positioned at center of the dish diffused to whole area. At low frequency, almost all particles sank on the bottom and hardly dispersed. (a) $f=0.5 \mathrm{~s}^{-1}$

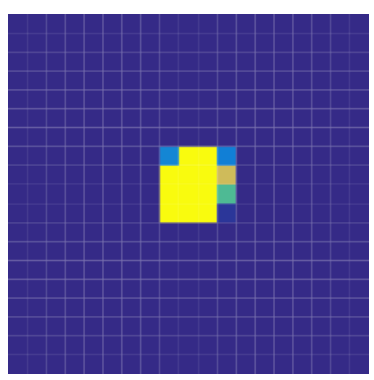

(c) $f=1.0 \mathrm{~s}^{-1}$

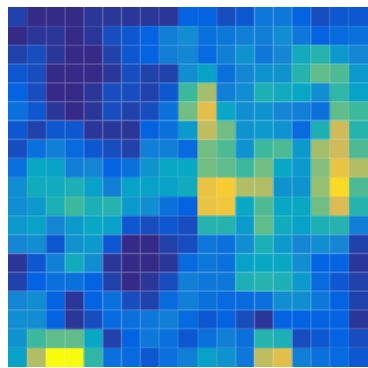

(e) $f=2.0 \mathrm{~s}^{-1}$

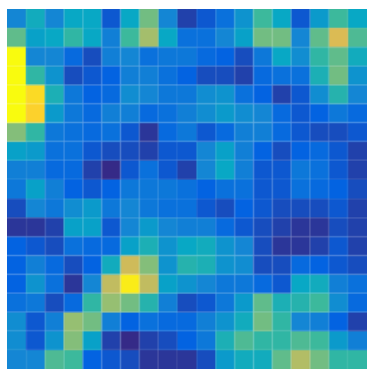

(b) $f=0.75 \mathrm{~s}^{-1}$

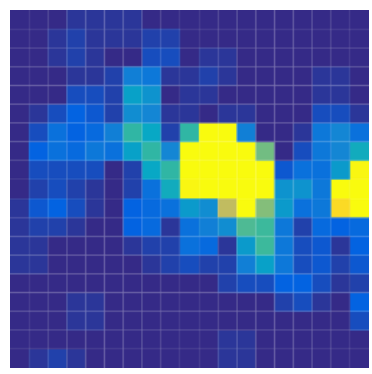

(d) $f=1.5 \mathrm{~s}^{-1}$

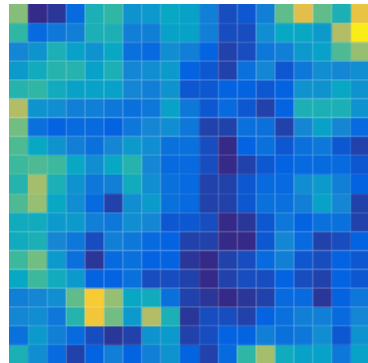

Abundance ratio

of the particles $(\%)$

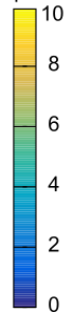

Fig. 3. Top view of particle distribution in shaking frequency $f=0.5,0.75,1.0,1.5$ and $2.0 \mathrm{~s}^{-1}$ (shaking method:MRS1)

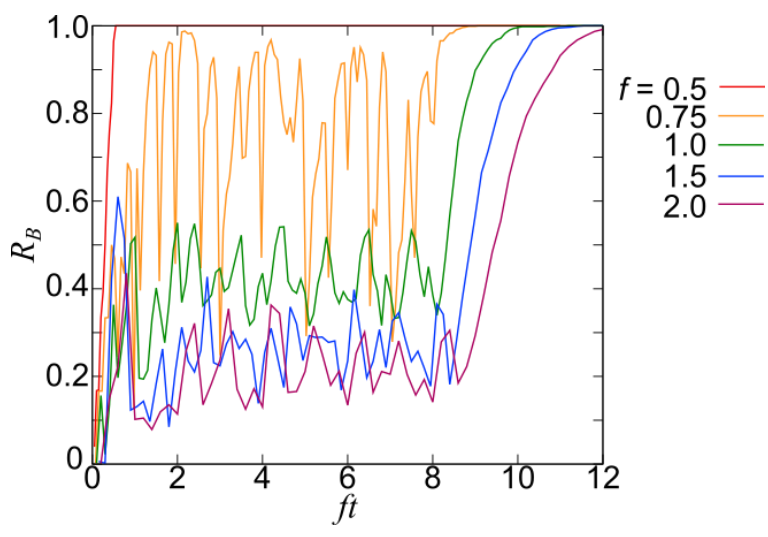

Fig. 4. $R_{\mathrm{B}}-f t$ variation in shaking frequency $f=0.5,0.75,1.0,1.5$ and, $2.0 \mathrm{~s}^{-1}$

To investigate the effect of shaking frequency in more detail, the authors evaluated the sedimentation by the ratio of particles that sank to the bottom. The 
sedimentation ratio was plotted as a function of nondimensional time, $f t$, in Fig. 4. $R_{\mathrm{B}}$ was defined as the follows.

$R_{\mathrm{B}}=\frac{\text { number of particles on the bottom }}{\text { total number of particles }(=990)}$

All particles in $f=0.5 \mathrm{~s}^{-1}$ immediately sank to the bottom after the beginning of the shaking. As the shaking frequency increased, $R_{\mathrm{B}}$ became smaller and particles were dispersed wider area. These results indicated that strong velocity fluctuations that lift particles from the bottom is required to disperse particles.

\section{Conclusion}

In this numerical research, the authors investigated that efficient shaking method to uniformly distribute particles in the reciprocal shaking bioreactor. The following results were obtained.

- In low shaking frequencies, almost all particles sank to the bottom and hardly dispersed.

- The shaking method that changes shaking direction at a given time interval was more effective to disperse particles than that moves on the same straight line.

These results indicated that strong fluctuations can lift particles from the bottom, and frequent change of flow direction makes distribution area wide.

As a future work, the effect of slowing down of the shaking and other shaking methods, e.g. wave bioreactor, will be investigated.

\section{Nomenclature}

\begin{tabular}{|c|c|}
\hline$A_{\mathrm{p}}$ & projected area $\left[\mathrm{m}^{2}\right]$ \\
\hline$C_{\mathrm{D}}$ & drag coefficient [-] \\
\hline$d_{s}$ & particle diameter $[\mathrm{m}]$ \\
\hline$F_{\mathrm{D}}$ & drag force $\left[\mathrm{kg} \cdot \mathrm{m} \cdot \mathrm{s}^{-2}\right]$ \\
\hline$F_{\mathrm{g}}$ & gravitational force $\left[\mathrm{kg} \cdot \mathrm{m} \cdot \mathrm{s}^{-2}\right]$ \\
\hline$f$ & shaking frequency $\left[\mathrm{m}^{-1}\right]$ \\
\hline$f t$ & non-dimensional time [-] \\
\hline$g$ & gravitational acceleration $\left[\mathrm{m} \cdot \mathrm{s}^{-2}\right]$ \\
\hline$m_{\mathrm{s}}$ & Mass of solid [kg] \\
\hline$n$ & normal vector $[-]$ \\
\hline$p$ & pressure $\left[\mathrm{kg} \cdot \mathrm{m}^{-1} \cdot \mathrm{s}^{-2}\right]$ \\
\hline$R_{\mathrm{a}}$ & shaking amplitude $[\mathrm{m}]$ \\
\hline$R_{\mathrm{B}}$ & sedimentation rate $[-]$ \\
\hline$R e_{\mathrm{p}}$ & Reynolds number of particle [-] \\
\hline$t$ & time $[\mathrm{s}]$ \\
\hline$U$ & velocity of shaking tank $\left[\mathrm{m} \cdot \mathrm{s}^{-1}\right]$ \\
\hline$v_{\mathrm{f}}$ & velocity of fluid $\left[\mathrm{m}^{-1} \mathrm{~s}^{-1}\right]$ \\
\hline$v_{s}$ & velocity of solid $\left[\mathrm{m} \cdot \mathrm{s}^{-1}\right]$ \\
\hline$\alpha$ & volume fraction of fluid [-] \\
\hline$\delta$ & delta function $\left[\mathrm{m}^{-1}\right]$ \\
\hline$\kappa$ & curvature of interface $[-]$ \\
\hline$\mu_{\mathrm{f}}$ & viscosity of fluid [Pa*s] \\
\hline$\mu_{\mathrm{s}}$ & viscosity of solid [Pa-s] \\
\hline$\rho_{\mathrm{f}}$ & density of fluid $\left[\mathrm{kg} \cdot \mathrm{s}^{-3}\right]$ \\
\hline$\rho_{\mathrm{f}}$ & density of solid $\left[\mathrm{kg} \cdot \mathrm{s}^{-3}\right]$ \\
\hline$\sigma$ & surface tension $\left[\mathrm{kg} \cdot \mathrm{s}^{-2}\right]$ \\
\hline
\end{tabular}

$\omega$

angular velocity $\left[\mathrm{rad} \cdot \mathrm{s}^{-1}\right]$

This research work is partially supported by the project of "Development of Cell Production and Processing Systems for Medical Research and Development, AMED, and by a Grantin-Aid for Scientific Research (B) (No. 15H4173) from the Ministry of Education, Culture, Sports, Science and Technology of Japan.

\section{References}

[1] K. Takahashi and S. Yamanaka: Induction of Pluripotent Stem Cells from Mouse Embryonic and Adult Fibroblast Cultures by Defined Factors, Cell 126, 2006, 663-676

[2] K. Takahashi, K. Tanabe, M. Ohnuki, M. Narita, T. Ichisaka K. Tomoda, and S. Yamanaka: Induction of Pluripotent Stem Cells from Adult Fibroblasts by Defined Factors, Cell 131, 2007, 861-872

[3] M. J. Evans and M. H. Kaufman: Establishment in culture of pluripotential cells from mouse embryos, Nature 292, 1981, 154-156

[4] T. Yamamoto, M. Yano, Y, Okano, and M, Kinooka: Numerical Investigation for the Movement of Cell Colonies in Bioreactors: Stirring and Orbital Shaking Tanks, J. Chem. Eng. Jpn. 51, 5, 2018, 423-430

[5] F. Moukalled, L. Mangani, and M. Darwish: The Finite Volume Method in Computational Fluid Dynamics, Springer International Publishing Switzerland, 2016

[6] L. Jing, C. Y. Kwok, Y. F. Leung, and Y. D. Sobral: Extended CFD-DEM for free-surface flow with multi-size granules, J. Numer. Anal. Meth. Geomech. 40, 2016, 62-79

[7] C. W. Hirt and B. D. Nichols: Volume of Fluid (VOF) Method for the Dynamics of Free Boundaries, J. Comput. Phys. 39, 1981, 201-225

[8] J. U. Brackbill, D. B. Kothe, and C. Zemach: A Continuum Method for Modeling Surface Tension, J. Comput. Phys. 100, 1992, 335-354

[9] Available online at https://www.openfoam.com 